\title{
Register van personen
}

Voorzover Indonesiërs na de in 1948/1949 ingevoerde spellingswijziging de oe-klank in hun naam met de letter -u- zijn gaan weergeven, is in dit register in het algemeen de eerdere spelling aangehouden. Namen van Japanners zijn in dit register in Engelse transcriptie weergegeven, onder toevoeging van een Nederlandse transcriptie waar daartoe aanleiding is.

Abdoelgani zie Roeslan Abdoelgani Abdoelkadir Widjojoatmodjo (1904-1992) $299,323,385-6,392,408,431,540,559$, $563,583,599,630$

Abdoh, Djalal 755

Abendanon, J.H. (1852-1925) 155, 157, 164,174

Abikoesno Tjokrosoejoso (1897-1968) 231, 240, 253, 259, 281, 284, 291, 298, $314,326,376,426,446,595,654$

Ageng, sultan (1631-1692) 42-3

Agoeng, sultan (1591-1645) 32-3, 41-2

Aidit, D.N. (1923-1965) 717

Albuquerque, Alfonso d' (1453-1515)

15-6

Ali Sastroamidjojo (1903-1976) 268, 272, $578,606,640,654,718,722,724-5,728$, 730

Alimin Prawirodirdjo (1889-1964) 198-9, 201, 594, 596

Alkadrie zie Hamid II

Amir Sjarifoeddin (1907-1948) 206, 219, 225, 230, 233, 240, 264, 272, 291, 305, $323,327,375-7,409,416,422,425-6$, $428,435,444,458,461-2,469,477,481$, $498,520,522,547,550-2,560,562,569$ 72, 594-7, 605

Anak Agoeng Gde Agoeng, Ide (1921-
2002) 513, 576, 583-4, 602, 610, 631, 633, 635, 641, 645, 647, 646, 661, 664, $666,668,670-3,695-6,715,719,723$, 742

Aponno, F.A. (1907-1983) 708

Aroeng Palaka (1634-1696) 41-2

Asbeck, F.M. van (1889-1968) 212

Assaat (1904-1976) 606, 672, 715

Attlee, C. (1883-1967) 389, 434, 527

Aung San (1915-1947) 278, 373, 390, 405, 526,577

Aziz, Andi Abdul 696-7, 700, 757

Bagoes Hadikoesoemo (1890-1954) 310, 345

Barentsz, Willem (1555-1597) 23

Bastian, A. (1826-1905) 105

Baud, J.C. (1789-1859) 85-6, 89-90, 92, 107, 148

Beel, L.J.M. (1902-1977) xviii, 357, 394, 434, 463-4, 471-2, 477, 507, 529, 53541, 547-8, 552, 554-5, 558, 564, 573, $581,585-7,598-603,609,629-31,633-6$, 638-9

Beerling, R.F. (1905-1979) 562

Beureuh, Daud (1906-1987) 725, 742

Bevin, E. (1881-1951) 434, 440

Boeng Tomo zie Soetomo 
Boer, F. de (1892-1976) 471, 473, 509, 524 Bonay, E. 755

Bosch, J. van den (1780-1844) 84-7, 91 Bosscha, K.A.R. (1865-1928) 143, 171 Both, Pieter $( \pm 1550-1615)$ 26, 65

Bouman, H.A. (1909-1968) 726-7

Bouman-van den Berg, M.S. (Mieke) (1907-1966) 727-9

Brooke, James (1803-1868) 89

Brooshooft, P. (1845-1921) 116

Bruins Slot, J.A.H.J.S. (1906-1972) 549, 759

Bunker, E. (1894-1984) 751-3

Burhanuddin zie Harahap

Buskes, J.J. (1899-1980) 441

Buurman van Vreeden, D.C. (1902-1964) $266,437-8,456,481,518,520,640$, 648-9

Bywater, Hector (1884-1940) 213

Camphuys, J. (1634-1695) 52, 104

Capellen, G.A.G.Ph. van der (1778-1848) 74-6, 81

Carpentier Alting, J.H. (1864-1929) 1734, 186, 190, 208, 241, 244, 407, 410

Chairoel Saleh (Chaerul Saleh) (19161968) 307, 333-4, 348-9, 359, 377, 381, $446,692,716-7,732,756$

Chasteleyn, Cornelis (1657-1714) 52

Christison, Ph. (1893-1993) 389-91, 393, 397-8, 400, 402, 405, 409, 412, 416-7, $429,435-7,447$

Chroesjtsjov, N.S. (1894-1971) 743

Churchill, W. (1874-1965) 256, 261, 301, 326, 370, 389

Clark Kerr, A.J. (1882-1951) 439, 449, 451-2, 459

Cochran, H.M. (1892-1973) 593-4, 599$601,603,625,638,656,716$

Coen, Jan Pieterszoon (1587-1629) 31-6, 43,65

Colijn, H. (1869-1944) 178-9, 193, 208-9, 211, 223-4, 236-7, 259, 367, 442, 453

Cramer, Ch.G. (1879-1976) 171-2, 236-7, 395

Critchley, T.K. (1916) 578, 580-1, 583, 593-4

Daalen, G.C.E. van (1863-1930) 119-20
Daendels, H.W. (1762-1818) 62, 71-7, 81

Darsono (1897-1971) 167, 186, 188, 199

Darwin, Charles (1809-1882) 105, 107

Dening, M. (1897-1977) 407, 423

Deventer, C.Th. van (1857-1915) 115-6

Dewantoro, Ki Hadjar zie Soewardi Soerjaningrat

Diah, B.M. (1917-1996) 333-4, 349, 377, 382

Diponegoro, prins (1785-1855) 80-3, 89, 193

Djajadiningrat, Achmad (1877-1943) 190, 208

Djajadiningrat, Hilman 574

Djajadiningrat, Hoesein (1886-1960) 174, 239, 243, 246, 298, 309, 314, 330, 409,

Djajadiningrat, Loekman (1891-1944) 243, 266, 299

Djojobojo, koning (regeerde van 1135 tot 1160) 9,56

Djuanda Kartawidjaja, R.H. (1911-1963) 732, 735-6, 738-40, 758

Doorman, Karel W.F.M. (1889-1942) 2623, 759

Douwes Dekker, E. (Multatuli) (18201887) 93-5, 113-4, 159

Douwes Dekker, E.F.E. (Setiaboedi) (1879-1950) 159-61, 163, 165, 180-1, $184-5,197-8,253,259,270,478$

Drees, W. (1886-1988) 368, 391, 395, 452, 454, 464, 547, 551, 558, 564, 584-5, 587, $592,598,600,602-3,606-7,629,631$, 633, 654, 658-9, 662-3, 672, 705, 707, $721,733-4,744,746$

DuBois, C. (1881-1960) 578, 580-1, 584, 593-4

Dubois, E. (1858-1940) 107

Duynstee, F.J.F.M. (1914-1981) 681, 748

Effendi, Roestam zie Roestam Effendi

Eisenhower, D.D. (1890-1969) 338, 731, 750

Erlangga (Airlangga), koning (regeerde van 1019 tot 1049) 7

Fatmawati (1923-1980) 306

Feuilletau de Bruyn, W.K.H. (1886-1972) $442,510,584-5,658,662$ 
Fock, D. (1858-1941) 177, 179, 183, 185, 203, 208

Gadjah Mada († 1364) 9

Gama, Vasco da (1469-1524) 15

Gandhi, Mohandas K. (Mahatma) (18691948) 182-3, 216, 219-21, 232, 364, 373, 526

Gani, A.K. (1905-1968) 230, 378, 478, 481, 511, 524, 550-2, 556

Gerbrandy, P.S. (1885-1961) 259, 299, 301, 336, 367-8, 510, 536, 549, 584, 658, 703

Gerretson, F.C. (1884-1958) 179, 192-3, 218, 441-2, 658

Goedhart, F.J. (1904-1990) 721

Goes van Naters, M. van der (1900-2005) 509,602

Graeff, A.C.D. de (1872-1957) 203-4, 207, 209, 212, 215-6, 218

Graham, F.P. (1886-1972) 561, 565, 578, 593

Groot, Hugo de (1583-1645) 28

Hadikoesoemo, Bagoes zie Bagoes Hadikoesoemo

Hagen, Steven van der (1563-1624) 26-7, 35

Hajam Woeroek, koning (1334-1389) 9

Hamengkoe Boewono IX, sultan (19121988) 428, 436, 478, 570, 641, 670-1, 715-8

Hamid II Alkadrie, sultan (1913-1978) 454, 466, 534, 583-4, 602, 609-10, 635, $641,654,671-2,695,742,756$

Harada, K. (1888-1947) 299, 309, 312, $323,326,328,332$

Harahap, Burhanuddin (1917-1987) 719, 723-4, 728, 738, 742

Hasanoedin, sultan († 1670) 40-1

Hasjim, Wahid zie Wahid Hasjim

Hassan, T.M. 378, 397

Hatta, Mohammad (1902-1980)

- afkomst en opleiding 194

Hazeu, G.A.J. (1870-1929) 155, 177, 190, 198

Heemskerk, Jacob van (1567-1607) 23, 26,35
Helfrich, C.E.L. (1886-1962) 262-4, 321, 405-6, 419-21, 437, 509-10, 657-8

Helsdingen, W.H. van (1888-1985) 234

Herremans, R. 578, 580

Heutsz, J.B. van (1851-1924) 118-21, 124, 131, 144, 157, 169, 178, 194

Heuven Goedhart, G.J. van (1901-1965) 441, 474, 549, 555

Heijden, K. van der (1826-1900) 99

Hinloopen Labberton, D. van (18741961) $159,169,171$

Hirohito, keizer (1901-1989) 310

Hirschfeld, H.M. (1899-1961) 672, 689, 692, 702, 704

Ho Chi Minh ( \pm 1890-1969) 373, 450-1, 525-6, 595

Hoëvell, W.R. van (1812-1879) 92-3, 103, 114-5, 148

Hogendorp, Dirk van (1761-1822) 69-70, 147

Houtman, Cornelis de (1550-1599) 23-4

Houtman, Frederik de (1554-1627) 24-5, 27,50

Idenburg, A.W.F. (1861-1935) 157, 161, 164, 170, 268

Idenburg, P.J.A. (1896-1976) 409, 431, $546,557,563$

Imam Bondjol zie Peto Sarif Imamura, H. (Imamoera) (1886-1968) 265, 267-8, 286, 294-5, 297, 299

Imhoff, G.W. van (1705-1750) 57

Iskandar Dinata, Oto (1897-1945) 216, 235, 305, 326, 345, 374, 376, 402

Iskandar Moeda, sultan (1590-1636) 40

Iwa Koesoema Soemantri (1899-1971) $239,274,359,376,384,426,461-2,490$, $572,595,718-9$

s'Jacob, H.L. (1906-1967) 650-1, 670

Jager, Herbert de (1636-1694) 50, 52

Jinnah, M.A. (1876-1948) 526

Jonge, B.C. de (1875-1958) 218, 220-1, 224, 236

Jonkman, J.A. (1891-1976) xv, 176, 203, 208, 211-2, 234, 240, 244, 255, 295, 4645, 467, 482-4, 508, 514, 521, 524, 535, $538-41,551,564,585,587,599,615$, 
$647,653-4,660,662$

Josselin de Jong, J.P.B. de (1886-1964) 441

Juliana, koningin (1909-2004) 223, 582, $658,672,706,729,744$

Junghuhn, F.W. (1809-1864) 105

Jungschläger, L.N.H. (1904-1956) 726-9, 733, 736

Kadt, J. de (1897-1988) 395, 422, 441, 489, 562, 604, 721

Kartini (1879-1904) 157

Kartosoewirjo, S.M. (1905-1962) 231, $273,572,627,692,724,727,742$

Kasimo, I.J. (1900-1986) 229, 235, 250, $305,375,425,570,605$

Kawilarang, A.E. (1920-2000) 742

Ken Angrok, koning (13e eeuw) 9

Kennedy, J.F. (1917-1963) 750-1, 753

Kennedy, R.F. (1925-1968) 751

Kern, J.H.C. (1833-1917) 107

Ketoet Poedja 378, 466

Kiai Modjo zie Modjo, kiai

Killearn, M.W.L. (1880-1964) 474, 478-81, 484

Kirby, R.C. (1904-2000) 561, 578

Kleffens, E.N. van (1894-1983) 245, 3401, 391, 440, 507, 555, 558, 602

Koch, D.M.G. (1881-1960) 240, 244

Kock, H.M. de (1779-1845) 82, 83

Koesoema Soemantri, Iwa zie Iwa Koesoema Soemantri

Koesoemo Joedo, prins (1882-1955) 131, 171, 208

Koesoemo Oetojo (1871-1953) 216, 2345, 309

Koets, P.J. (1901-1995) 212, 244, 466, 479, 546, 635

Koiso, K. (1880-1950) 325-7, 331-2

Kol, H.H. van (1852-1925) 115-7

Kruls, H.J. (1902-1975) 509

Kuyper, A. (1837-1920) 112, 115-6, 178

Lamping, A.Th. (1893-1970) 704, 721

Latuharhary, J. (1900-1959) 345, 378

Lawson, J.J. 389-90, 393, 398

Lieftinck, P. (1902-1989) 434, 465, 482

Leimena, J. (1905-1977) 425, 446, 551,
$570,592,640,654,698-9$

Limburg Stirum, J.P. van (1873-1948) 170-3, 177, 179, 203, 270

Linschoten, Jan Huyghen van (15631611) 21,23

Lodewijk Napoleon, koning (1778-1846) 71

Logan, J.R. († 1869) 105

Logemann, J.H.A. (1892-1969) 212, 338, 368-9, 390, 392-4, 403, 407-9, 430, 433$4,439,442-3,449,452,454-6,464,654$

Lovink, A.H.J. (1902-1999) 264, 266, 639, 648-50, 672

Luns, J.M.A.H. (1911-2002) 721, 723, 733-4, 743-5, 747-50, 752

Maarseveen, J.H. van (1894-1951) 634, 640, 648, 653-4, 662-4, 680, 706-7, 757

MacArthur, D. (1880-1964) 233, 261, 278, 321-3, 337, 339-40, 370, 525, 542

Maeda, T. (1898-1977) 289, 327, 333, 3479, 397

Magelhaes, Fernando de (1480-1521) 16

Malik, Adam (1917-1984) 333-4, 377, $381,383,427,462,751-3$

Mallaby, A.W.S. (1899-1945) 415-6

Mangoenkoesoemo, Tjipto zie Tjipto Mangoenkoesoemo

Mansoer, kiai (1896-1946) 280, 297-8, 305, 308, 310, 316

Mansoer, Tengkoe (1897-1955) 574, 583, 701

Manuhutu, J.H. 698-9

Manusama, J.A. (1910-1995) 698, 705

Mao Tse-Tung (1893-1976) 595

Maramis, A.A. (1897-1977) 376

Matoelesia, Thomas (1783-1817) 78, 698

Maurits, prins (1567-1625) 25

Meyer Ranneft, J.W. (1887-1968) 208, $242,442,510,658,662$

Miyoshi, S. (Mijosji) 289

Modjo, kiai $( \pm 1792-1849)$ 82-3

Moeis, Abdoel (1890-1959) 164, 169, 171, $180,186-9$

Moelia, T.S.G. (1896-1966) 229, 240, 244, 251-2, 291, 298, 426

Moeso (1897-1948) 198-9, 201-2, 594-7, 676,714 
Monginsidi, W. (1925-1949) 516-7, 519, 650

Mook, H.J. van (1894-1965)

- afkomst en opleiding 271

Mountbatten, L. (1900-1979) 321-2, 340, 370-2, 379, 386, 389-91, 397-8, 405-6, 408, 410, 412, 416, 418, 430, 447, 450, 458-9

Multatuli zie E. Douwes Dekker

Mussert, A.A. (1894-1946) 366, 409, 510

Muzakkar, Kahar (1919-1965) 725, 742

Nadjamoeddin Daeng Malewa 466-7, 512-3, 576

Nagano, J. 332, 361, 380-4, 386

Napoleon, keizer (1769-1821) 71-3

Nasoetion, A.H. (1918-2000) 469, 570, 590, 605, 625, 630, 717-9, 736, 738-41, 743, 748-9

Nasser, G.A. (1918-1970) 719

Natsir, M. (1908-1993) 444, 446, 478, 570, 592, 701, 704-5, 707, 715-7, 720, 738, 742

Neck, Jacob van (1564-1638) 24, 26

Nederburgh, S.C. (1762-1811) 61, 69

Neher, L. (1889-1967) 538, 564, 587, 602

Nehru, Jawaharlal (1889-1964) 195, 373, 430, 474, 526-7, 534, 556, 606-7, 719

Nishijima, S. (Nisjidjima) 289, 327, 348-9

Noor, Tadjoeddin zie Tadjoeddin Noor

Noort, Olivier van (1558-1627) 24

Noto Soeroto (1888-1951) 175, 194

Oerip Soemohardjo (1893-1948) 404, 520, 570, 671

Oldenbarnevelt, Johan van (1547-1619) 21,25

Oltmans, W. (1925-2004) 735, 748

Oyen, L.H. van (1889-1953) 321, 419-21, 437

Palar, L.N. (1900-1981) 497, 555, 561, 605

Pamontjak, M.N. 272, 497

Pati Oenoes, prins († 1521) 15

Patterson, W.R. (1893-1954) 384-6, 405

Pattimoera zie Matoelesia

Peekema, W.G. (1899-1967) 303

Peto Sarif (Imam Bondjol) (1772-1864)
89

Pinke, A.S. (1894-1966) 437, 519-20, 558, 561, 657

Plas, Ch.O. van der (1891-1977) 212, 244, 264, 266, 299, 321, 324, 326, 337, 339, 344, 367-8, 372, 384-6, 389-94, 405, 407, $409,431,437,494,574$

Poedja zie Ketoet Poedja

Poeradiredja, Adil 575, 583-4

Poetiray, E. (1918) 441

Poll, M.J.M. van (1881-1948) 443, 454-5, $471,481,508,524,532,543,546,552$, 555, 558

Polo, Marco (1254-1323) 14

Poorten, H. ter (1887-1968) 262-3, 266-7, 276-7

Pordenone, Oderico de (1286-1331) 14

Prawiradirdjo (Sentot) (1808-1854) 82-3, 89, 93

Prawiranegara, Sjafroedin zie Sjafroedin Prawiranegara

Princen, J.C. (Poncke) (1925-2002) 625, 758

Pringgodigdo, A.K. (1906-1961) 452, 454, 606

Quay, J.E. de (1901-1985) 744, 746-8

Quezon, M.L. (1878-1944) 233-4, 525

Quisling, V. (1887-1945) 366

Radjiman Wedioningrat (1879-1952) 331, 341, 344-5

Raffles, Thomas Stamford (1781-1826) 72-7, 80-1, 103-4

Randwijk, H. van (1909-1966) 441, 474, 554

Ratoelangie, G.S.S.J. (1890-1949) xv, xix, 175-6, 229, 235, 240-1, 244, 253, 271, $305,310,345,378,457,466,512,514$, $516,578,604,647$

Razak, N. 726-7

Reael, Laurens (1583-1637) 31, 35-6

Reinwardt, C.G.C. (1773-1854) 105

Resink, G.J. (1911-1997) 562

Rikhye, I.J. (1920-2007) 755

Ringers, J.A. (1885-1965) 482, 538

Rivai, Abdoel (1871-1933) 154, 159, 171, 198, 270 
Rizal, José (1861-1896) 158

Roem, Mohammad (1908-1983) 231, 478, $481,484,511,524,578,580-1,606,622$, 636, 638-42, 645, 647, 650, 654, 663, 670-2, 691, 704, 707, 715-6, 742

Roeslan Abdoelgani (1914-2005) 334, 733

Roestam Effendi (1905-1979) 223, 409, 497

Romme, C.P.M. (1896-1980) 453, 463-5, 508-9, 529, 537, 599, 602, 636, 653-4, 664, 720-1

Roorda van Eysinga, S.E.W. (1825-1887) 93, 135

Roosevelt, F.D. (1882-1945) 256, 261, 245, 278, 301-2, 321, 326

Roijen, J.H. van (1905-1991) xviii, 391, $434,452,605-7,622,631,636,638-43$, 645, 647-8, 650, 653-4, 663-4, 691, 751, 753

Rumphius, G. (1628-1702) 50, 52

Rijkens, P.C. (1888-1965) 720, 722, 734-5, 748

Saleh, Chairoel zie Chairoel Saleh

Salim, Agoes (1884-1954) xv, xix, 180, 186-8, 198, 206, 216, 231, 236, 305, 377, 409-10, 446, 477, 534, 548, 550-1, 556, $570,592,606,610,631,643$

Sanders, P. (1912) 555

Sartono (1900-1968) xix, 204, 207, 215, 217, 219, 230, 236, 297, 326, 426, 714

Sassen, E.M.J.A. (1911-1995) 585, 592-3, 598-2, 609-10, 628-9, 634

Sastroamidjojo, Ali zie Ali Sastroamidjojo

Schermerhorn, W. (1894-1977) 356-8, 368-70, 388, 391-4, 407, 410, 419, 434, 442-3, 452-6, 460, 463-5, 471, 476, 4789, 481, 483-4, 521-4, 532, 535, 537, 541, $543,546,551-2,555,558,562,664,709$ 10,737

Schilling, W. (1890-1958) 266, 397, 401, $420,431,437,455$

Schmidt, H.C.J.G. 726-9, 758

Schmutzer, J.I.J.M. (1882-1946) 338

Semaoen (1899-1971) 166-7, 172, 186-8, 195, 198-9, 271
Senopati († 1601) 11, 42

Sentot zie Prawiradirdjo

Setiaboedi zie E.F.E. Douwes Dekker

Setiadjit (1907-1948) 368, 441, 454, 478, 497, 550-1, 569

Siliwangi, koning (14e eeuw) 9, 469

Simatoepang, T.B. (1920-1990) 630, 643, 654, 717-8

Sjafroeddin Prawiranegara (1911-1989) xix, 446, 478, 570, 604, 630, 636, 640, $642,715,738,742$

Sjahrir (1909-1966) xviii, xix, 195, 217, 219, 227, 239, 250, 261, 272, 297, 306, $327,329,333-4,344-5,347,356-7,375$, $377,384,395,421-30,432-6,439,441-6$, 449-51, 453, 458-62, 469-70, 473-4, 47781, 483-4, 500, 522-4, 532-5, 539, 545, 549-50, 554, 556-7, 572, 606, 665, 668, $714,716,718,742$

Sjarifoeddin, Amir zie Amir Sjarifoeddin

Slotemaker de Bruïne, G.H. (1899-1976) 441

Sneevliet, H.J.F.M. (1888-1942) 165-7, 181, 186, 215, 223

Snouck Hurgronje, C. (1857-1936) 107, 117-9, 121-2, 131, 174, 190, 192

Soebandrio (1914-2004) 747, 749, 753

Soebardjo, Achmad (1896-1978) 280, $289,327,333,345,348,359,376,384$, $409,426,461-2,572,595,716$

Soedarsono (generaal-majoor) 461-2, 572,595

Soedarsono (minister) (1911-1976) 452

Soedirman (1912-1950) 428, 445, 461-2, 470, 480-1, 520-1, 523-4, 570, 603, 605, $628,630,636,640,642,646,649,756$

Soeharto (1921-2008) 628, 751, 759

Soejono (1886-1943) 243, 252, 266, 299, 301-3

Soekarni (1916-1971) 273, 307, 333-4, 348-9, 359, 381, 383, 427, 446, 595

Soekarno (1901-1970)

- afkomst en opleiding 198

Soekawati, T.G.R. (1899-1979) 216, 237, 251, 466, 512-3, 576, 696-7, 701

Soekiman Wirjosandjojo (1898-1974) xix, 206, 231, 305, 425, 569, 605, 630, 654, $715-7,720$ 
Soepomo (1903-1958) 252, 289, 298, 309, $326,330,341-2,376,607,654,701,720$

Soeriadarma, S. (1912-1975) 606

Soeripno (1920-1948) 580, 595, 605

Soeropati (1660-1706) 55

Soetardjo Kartohadikoesoemo (18901976) 216, 234-9, 241, 251, 255-6, 305, $309,345,375,673$

Soetomo (dokter) (1888-1938) 159, 176, 197, 203, 206, 216-7, 219-20, 229-30, $239,253,270,384$

Soetomo (Boeng Tomo) (1920-1981) 417, 470, 511, 706, 720

Soewandi (1899-1964) 452-3

Soewardi Soerjaningrat (Ki Hadjar Dewantoro) (1889-1959) xv, xix, 159, 161-4, 175-6, 181, 183-5, 217, 220-1, 244-5, 270, 273, 280, 297-8, 305, 308, $326,359,376,426,604,673$

Soumokil, C. (1905-1966) 696-8, 705, 742 Speelman, Cornelis (1628-1684) 41-3 Spit, H.J. (1886-1967) 294, 380-1, 399 Spoor, S.H. (1902-1949) xviii, 266, 325, 393, 421, 437-8, 454-5, 513, 518, 523, 529-30, 537, 539, 541-2, 551-3, 555, 558, 564-5, 570, 587-8, 599, 601, 603, 612, $626,629-30,636-9,700$

Stikker, D.U. (1897-1979) 585, 594, 599, 601-2, 610, 628, 654, 707, 721

Sumual, H.N. Ventje (1923) 732, 742

Tadjoeddin Noor 311, 512-4, 516, 518, 575

Tagore, Rabindranath (1861-1941) 157, 182, 185

Tahija, J.W. (1916-2002) 454, 513, 700

Tan Malaka, Ibrahim (1897-1949) 184-5, 188, 195, 198-202, 329, 333, 342, 383-4, 427, 444-6, 458, 461-2, 572, 595-7, 627, $640,692,714$

Tantri, K'tut (pseudoniem van Muriel Pearson) (1899-1997) 418

Tasman, Abel (1602-1659) 50

Terauchi, H. (Teraoetsji) (1879-1946) 297, 310, 344-5, 372

Teukoe Oemar (1854-1899) 117-8, 403

Thamrin, M.H. (1894-1941) 153, 203, 216-20, 225, 228-9, 233, 235, 240-1, 244-
$5,251-3,272,384$

Tjarda van Starkenborgh Stachouwer, A.W.L. (1888-1978) 236, 238, 245, 250, 253, 255-9, 261, 263-4, 266-7, 292, 3934, 407, 409

Tjipto Mangoenkoesoemo (1886-1943) 159, 161, 163, 166, 171, 180-3, 198, 203, 205, 213, 225, 236, 239, 245, 250, 270, 274,384

Tjoet Nja Din (1850-1908) 119-20

Tjokroaminoto, Oemar Said (1882-1934) 163-5, 171-2, 180, 186-8, 197-8, 203, 205-6, 228, 231, 236

Tojo, H. (Toodjo) (1884-1948) 308-10, 312,325

Treub, M.W.F. (1858-1931) 179, 190, 192, 211

Trimoerti (1912-2009) 273

Trip, L.J.A. (1876-1947) 208-9

Troenodjojo, prins (1640-1680) 42

Truman, H.S. (1884-1972) 370

U Thant (1909-1974) 751, 753-5

Uyl, J.M. den (1919-1987) 554, 562

Van der Post, L. (1906-1996) 276, 380-1, 385-6, 389

Van Zeeland, P. (1893-1973) 561, 578

Verdoorn, J.A. (1903-1991) 441

Veth, P.J. (1814-1895) 105

Victoria, koningin (1819-1901) 110

Visman, F.H. 252

Vollenhoven, C. van (1874-1933) 107, 190

Vredenburch, H.F.L.K. van (1905-1981) 563,581

Wahid Hasjim, kiai (1914-1953) 314, 330, 345

Wallace, A.R. (1823-1913) 105, 107

Wavell, A. (1883-1950) 262-3

Welles, S. (1892-1961) 301

Welter, Ch.J.I.M. (1880-1972) 208, 237-9, 242, 245, 254, 259, 442-3, 464-5, 510, $537,584-5,658,662,669,680$

Westerling, R.P.P. (1919-1987) 517-8, 691$5,697,725,756$

Wikana (1914-1965) 273, 327, 333-4, 347- 
$8,359,381,444,446,596$

Wilhelmina, koningin (1880-1962) 112, $114,169,171,238-9,244-5,255,299$, 302-3, 364, 366, 368-9, 388, 410, 466, $473,475,509,557,582,617$

Willem I, koning (1772-1843) 74, 76, 84, 86-7

Willem III, koning (1817-1890) 93, 112

Willem IV, prins (1711-1751) 59

Willem V, prins (1748-1806) 62, 74

Willem van Oranje (de Zwijger), prins (1533-1584) 21, 25

Wilopo (1908-1981) 716-8, 722
Winkelman, H.G. (1876-1952) 510, 658

Wiranatakoesoema (1888-1965) 216, 221, 229, 234, 359, 375-6, 459, 575, 694

Wiwoho Poerbohadidjojo 250-2

Wongsonegoro (1897-1978) 384

Yamamoto, M. (Jamamoto) 360, 380, 384 Yamin, Muhammad (1903-1962) xix, 206-7, 219, 230, 233, 245, 297, 309, 326, $333,341,377,446,462,572,595,654$, $706,716,718,720$ 